TRANSACTIONS OF THE

AMERICAN MATHEMATICAL SOCIETY

Volume 358, Number 1, Pages 391-402

S 0002-9947(05)03827-4

Article electronically published on July 26, 2005

\title{
A CONNES-AMENABLE, DUAL BANACH ALGEBRA NEED NOT HAVE A NORMAL, VIRTUAL DIAGONAL
}

\author{
VOLKER RUNDE
}

\begin{abstract}
Let $G$ be a locally compact group, and let $\mathcal{W} \mathcal{A P}(G)$ denote the space of weakly almost periodic functions on $G$. We show that, if $G$ is a [SIN]-group, but not compact, then the dual Banach algebra $\mathcal{W} \mathcal{A} \mathcal{P}(G)^{*}$ does not have a normal, virtual diagonal. Consequently, whenever $G$ is an amenable, non-compact [SIN]-group, $\mathcal{W} \mathcal{A} \mathcal{P}(G)^{*}$ is an example of a Connesamenable, dual Banach algebra without a normal, virtual diagonal. On the other hand, there are amenable, non-compact, locally compact groups $G$ such that $\mathcal{W} \mathcal{A P}(G)^{*}$ does have a normal, virtual diagonal.
\end{abstract}

\section{INTRODUCTION}

In [Joh 2], B. E. Johnson showed that a locally compact group $G$ is amenable if and only if its group algebra $L^{1}(G)$ has vanishing first order Hochschild cohomology with coefficients in dual Banach $L^{1}(G)$-bimodules. Consequently, he called a Banach algebra satisfying this cohomological triviality condition amenable. Soon thereafter, Johnson gave a more intrinsic characterization of the amenable Banach algebras in terms of approximate and virtual diagonals (Joh 3]).

For some classes of Banach algebras, amenability in the sense of [Joh 2] is too strong to allow for the development of a rich theory: it follows from work by S. Wassermann (Was 1]), for example, that a von Neumann algebra is amenable if and only if it is subhomogeneous. This indicates that the definition of amenability should be modified when it comes to dealing with von Neumann algebras.

A variant of Johnson's definition that takes the dual space structure of a von Neumann algebra into account was introduced in $\mathrm{J}-\mathrm{K}-\mathrm{R}$, but is most commonly associated with A. Connes' paper Con 1]. Following A. Ya. Helemskiǔ ([Hel]), we shall refer to this variant of amenability as Connes-amenability. As it turns out, Connes-amenability is equivalent to several other important properties of von Neumann algebras, such as injectivity and semidiscreteness ( $\overline{B-P}$, Con 1, Con 2], [E-L, Was 2]; see [Run 2, Chapter 6] for a self-contained exposition). Like the amenable Banach algebras, the Connes-amenable von Neumann algebras allow for an intrinsic characterization in terms of diagonal type elements: a von Neumann algebra is Connes-amenable if and only if it has a normal, virtual diagonal ([Eff $]$ ).

Received by the editors October 26, 2003 and, in revised form, June 1, 2004.

2000 Mathematics Subject Classification. Primary 46H20; Secondary 22A15, 22A20, 43A07, 43A10, 43A60, 46H25, 46M18, 46M20.

Key words and phrases. Locally compact groups, Connes-amenability, normal, virtual diagonals, weakly almost periodic functions, semigroup compactifications, minimally weakly almost periodic groups.

(C)2005 American Mathematical Society Reverts to public domain 28 years from publication 
The notions of Connes-amenability and normal, virtual diagonals make sense not only for von Neumann algebras, but for a larger class of Banach algebras called dual Banach algebras in Run 1]. Examples of dual Banach algebras, besides von Neumann algebras, are - among others - the measure algebras $M(G)$ of locally compact groups $G$. As for von Neumann algebras, amenability in the sense of [Joh 2 ] turns out to be too restrictive a concept for measure algebras: the algebra $M(G)$ is amenable if and only if $G$ is discrete and amenable $([\mathrm{D}-\mathrm{G}-\mathrm{H}]$. In Run 3, however, the author showed that $M(G)$ is Connes-amenable if and only if $G$ is amenable, and in Run 4, he also proved that these conditions are also equivalent to $M(G)$ having a normal, virtual diagonal.

It is not hard to see that any dual Banach algebra with a normal, virtual diagonal must be Connes-amenable: as is observed in [C-G], the argument from [Eff] for von Neumann algebras carries over almost verbatim. The converse, however, has been open so far ([Run 2, Problem 23]).

Besides $M(G)$ there are other dual Banach algebras associated with a locally compact group $G$. One of them is $\mathcal{W} \mathcal{A} \mathcal{P}(G)^{*}$, where $\mathcal{W} \mathcal{A} \mathcal{P}(G)$ denotes the weakly almost periodic functions on $G$. It is easy to see that $\mathcal{W} \mathcal{A P}(G)^{*}$ is Connes-amenable if $G$ is amenable; the converse is also true, but not as straightforward (Run 5]). If $G$ is compact, $\mathcal{W} \mathcal{A P}(G)^{*}=M(G)$ has a normal, virtual diagonal, and in Run 5, the author made the - as will become apparent: uneducated - guess that $\mathcal{W} \mathcal{A} \mathcal{P}(G)^{*}$ has a normal, virtual diagonal if and only if $G$ is compact.

In the present paper, we shall confirm this conjecture for [SIN]-groups. Consequently, whenever $G$ is an amenable [SIN]-group that fails to be compact, the dual Banach algebra $\mathcal{W} \mathcal{A} \mathcal{P}(G)^{*}$ is Connes-amenable, but has no normal, virtual diagonal. On the other hand, we shall see that $\mathcal{W} \mathcal{A P}(G)^{*}$ does indeed have a normal, virtual diagonal if $G$ is amenable and minimally weakly almost periodic in the sense of Chou 1]. Since there are such groups which fail to be compact, this shows that our conjecture from [Run 5] cannot be true in the generality stated there.

\section{Connes-Amenability And nORmal, virtual diagonals}

This section is preliminary in character: we briefly recall the definition of a dual Banach algebra along with the notions of Connes-amenability and of a normal, virtual diagonal.

Given a Banach algebra $\mathfrak{A}$ and a Banach $\mathfrak{A}$-bimodule $E$, the dual space $E^{*}$ of $E$ becomes a Banach $\mathfrak{A}$-bimodule in its own right via

$$
\langle x, a \cdot \phi\rangle:=\langle x \cdot a, \phi\rangle \quad \text { and } \quad\langle x, \phi \cdot a\rangle:=\langle a \cdot x, \phi\rangle \quad\left(a \in \mathfrak{A}, \phi \in E^{*}, x \in E\right) ;
$$

in particular, the dual space $\mathfrak{A}^{*}$ of $\mathfrak{A}$ is a Banach $\mathfrak{A}$-bimodule. Modules of this kind are referred to as dual Banach modules.

The following definition was introduced in Run 1.

Definition 1.1. A Banach algebra $\mathfrak{A}$ is called a dual Banach algebra if there is a closed submodule $\mathfrak{A}_{*}$ of $\mathfrak{A}^{*}$ such that $\mathfrak{A}=\left(\mathfrak{A}_{*}\right)^{*}$.

Remarks. 1. Equivalently, a Banach algebra $\mathfrak{A}$ is dual if it is a dual Banach space such that multiplication is separately continuous in the $w^{*}$-topology.

2. In general, the predual space $\mathfrak{A}_{*}$ in Definition 1.1 need not be unique, but will always be unambiguous from the context. 
Examples. 1. Every von Neumann algebra is a dual Banach algebra.

2. The measure algebra $M(G)$ of a locally compact group $G$ is a dual Banach algebra (with predual $\mathcal{C}_{0}(G)$ ).

3. If $E$ is a reflexive Banach space, then $\mathcal{B}(E)$ is a dual Banach algebra with predual $E \hat{\otimes} E^{*}$, where $\hat{\otimes}$ denotes the projective tensor product of Banach spaces.

4. The bidual of every Arens regular Banach algebra is a dual Banach algebra.

The following definition introduces a notion of amenability for dual Banach algebras that takes the dual space structure into account:

Definition 1.2. Let $\mathfrak{A}$ be a dual Banach algebra.

(a) A dual Banach $\mathfrak{A}$-bimodule $E$ is called normal if the maps

$$
\mathfrak{A} \rightarrow E, \quad a \mapsto\left\{\begin{array}{c}
a \cdot x \\
x \cdot a
\end{array}\right.
$$

are $w^{*}$-continuous for each $x \in E$.

(b) $\mathfrak{A}$ is called Connes-amenable if every $w^{*}$-continuous derivation from $\mathfrak{A}$ into a normal, dual Banach $\mathfrak{A}$-bimodule is inner.

Remarks. 1. For a von Neumann algebra, Connes-amenability is equivalent to a number of important properties, such as injectivity and semidiscreteness; see Run 2, Chapter 6] for a relatively self-contained account and for further references.

2. The measure algebra $M(G)$ of a locally compact group $G$ is Connes-amenable if and only if $G$ is amenable ([Run 3] ).

Let $\mathfrak{A}$ be a Banach algebra. Then $\mathfrak{A} \hat{\otimes} \mathfrak{A}$ is a Banach $\mathfrak{A}$-bimodule via

$$
a \cdot(x \otimes y):=a x \otimes y \quad \text { and } \quad(x \otimes y) \cdot a:=x \otimes y a \quad(a, x, y \in \mathfrak{A}),
$$

so that the multiplication map

$$
\Delta: \mathfrak{A} \hat{\otimes} \mathfrak{A} \rightarrow \mathfrak{A}, \quad a \otimes b \mapsto a b
$$

becomes a homomorphism of Banach $\mathfrak{A}$-bimodules. Let $\mathfrak{A}$ be a dual Banach algebra with predual $\mathfrak{A}_{*}$, and let $\mathcal{B}_{\sigma}^{2}(\mathfrak{A}, \mathbb{C})$ denote the bounded, bilinear functionals on $\mathfrak{A} \times \mathfrak{A}$ which are separately $w^{*}$-continuous, which form a closed submodule of $(\mathfrak{A} \hat{\otimes} \mathfrak{A})^{*}$. Since $\Delta^{*}$ maps $\mathfrak{A}_{*}$ into $\mathcal{B}_{\sigma}^{2}(\mathfrak{A}, \mathbb{C})$, it follows that $\Delta^{* *}$ drops to an $\mathfrak{A}$ bimodule homomorphism $\Delta_{\sigma}: \mathcal{B}_{\sigma}^{2}(\mathfrak{A}, \mathbb{C})^{*} \rightarrow \mathfrak{A}$.

We define:

Definition 1.3. A normal, virtual diagonal for a dual Banach algebra $\mathfrak{A}$ is an element $\mathrm{M} \in \mathcal{B}_{\sigma}^{2}(\mathfrak{A}, \mathbb{C})^{*}$ such that

$$
a \cdot \mathrm{M}=\mathrm{M} \cdot a \quad \text { and } \quad a \Delta_{\sigma} \mathrm{M}=a \quad(a \in \mathfrak{A}) .
$$

Remarks. 1. Every dual Banach algebra with a normal, virtual diagonal is Connesamenable ( $\mathrm{C}-\mathrm{G}]$, but actually already [Eff] $)$.

2. A von Neumann algebra is Connes-amenable if and only if it has a normal, virtual diagonal ([Eff] $)$.

3. The same is true for the measure algebras of locally compact groups ( Run 3] and Run 4]).

As we shall see in this paper, there are Connes-amenable, dual Banach algebras which do not have a normal, virtual diagonal. 


\section{The Banach algebra $\mathcal{W} \mathcal{A} \mathcal{P}(G)^{*}$ and the semigroup $G_{\mathcal{W} \mathcal{A}}$}

By a semitopological semigroup, we mean a semigroup $S$ equipped with a Hausdorff topology such that multiplication is separately continuous. If $S$ is locally compact, the measure space $M(S) \cong \mathcal{C}_{0}(S)^{*}$ can be turned into a Banach algebra via

$$
\langle f, \mu * \nu\rangle:=\int_{S} \int_{S} f(s t) d \mu(s) d \nu(t) \quad(\mu, \nu \in M(S)) .
$$

(Even though multiplication in $S$ need not be jointly continuous, the product integral on the right-hand side of (1) does always exist and is independent of the order of integration; see [Joh 1].) Note, that even though $M(S)$ is a Banach algebra which is a dual Banach space, it need not be a dual Banach algebra in the sense of Definition 1.1 this is due to the fact that $\mathcal{C}_{0}(S)$ need not be translation invariant. However, $M(S)$ is a dual Banach algebra if $S$ is compact or a group.

Our first proposition is likely to be well known, but since we could not locate a reference, we include a proof:

Proposition 2.1. Let $S$ be a locally compact, semitopological semigroup. Then the following are equivalent:

(i) $S$ has an identity.

(ii) $M(S)$ has an identity of norm one.

Proof. (i) $\Longrightarrow$ (ii): If $S$ has an identity element, say $e$, then the point mass $\delta_{e}$ is an identity for $M(S)$ and trivially has norm one.

(ii) $\Longrightarrow($ i): Suppose that $M(S)$ has an identity element, say $\epsilon$, such that $\|\epsilon\|=1$; in particular,

$$
\delta_{s} * \epsilon=\delta_{s}=\epsilon * \delta_{s} \quad(s \in S)
$$

holds. It is straightforward that $\epsilon$ has to be an $\mathbb{R}$-valued measure. Let $\epsilon^{+}, \epsilon^{-} \epsilon$ $M(S)$ be the Jordan decomposition of $\epsilon$, i.e. positive measures such that $\epsilon=\epsilon^{+}-\epsilon^{-}$ and $1=\left\|\epsilon^{+}\right\|+\left\|\epsilon^{-}\right\|$. Fix $s \in S$. Then $\delta_{s} * \epsilon^{+}$and $\delta_{s} * \epsilon^{-}$are positive measures such that $\delta_{s}=\delta_{s} * \epsilon^{+}-\delta_{s} * \epsilon^{-}$and

$$
\left\|\delta_{s}\right\|=1=\left\|\epsilon^{+}\right\|+\left\|\epsilon^{-}\right\| \geq\left\|\delta_{s} * \epsilon^{+}\right\|+\left\|\delta_{s} * \epsilon^{-}\right\| \geq\left\|\delta_{s} * \epsilon\right\|=\left\|\delta_{s}\right\|=1 .
$$

The uniqueness of the Jordan decomposition of $\delta_{s}$ thus yields that $\delta_{s} * \epsilon^{+}=\delta_{s}$. Analogously, one sees that $\epsilon^{+} * \delta_{s}=\delta_{s}$. Hence, (2) still holds true if we replace $\epsilon$ by $\epsilon^{+}$.

We shall see that the existence of a positive measure $\epsilon \in M(S)$ satisfying (2) already necessitates $S$ to have an identity.

Fix $s \in S$, and assume that there is $t \in \operatorname{supp}(\epsilon)$ such that $s t \neq s$. We may choose a non-negative function $f \in \mathcal{C}_{0}(S)$ such that $f(s)=0$ and $f(s t)>0$. We obtain that

$$
0<\int_{S} f(s t) d \epsilon(t)=\left\langle f, \delta_{s} * \epsilon\right\rangle=\left\langle f, \delta_{s}\right\rangle=f(s)=0,
$$

which is nonsense. Consequently, st $=s$ holds for all $t \in \operatorname{supp}(\epsilon)$; an analogous argument shows that $t s=s$ for all $t \in \operatorname{supp}(\epsilon)$. Since $s \in S$ was arbitrary, it follows that every element of $\operatorname{supp}(\epsilon)$ is an identity for $S$. 
Let $S$ be any semitopological semigroup, and let $f: S \rightarrow \mathbb{C}$. For $s \in S$, we define the left translate $L_{s} f$ of $f$ by $s$ through

$$
\left(L_{s} f\right)(t):=f(s t) \quad(t \in S) .
$$

Let $\mathcal{C}_{\mathrm{b}}(S)$ denote the commutative $C^{*}$-algebra of bounded, continuous functions on $S$.

Definition 2.2. Let $S$ be a semitopological semigroup. A bounded, continuous function $f \in \mathcal{C}_{\mathrm{b}}(S)$ is called weakly almost periodic if $\left\{L_{s} f: s \in S\right\}$ is relatively compact in the weak topology on $\mathcal{C}_{\mathrm{b}}(S)$.

For any semitopological semigroup $S$, let

$$
\mathcal{W} \mathcal{A P}(S):=\left\{f \in \mathcal{C}_{\mathrm{b}}(S): f \text { is weakly almost periodic }\right\} .
$$

Our reference for almost periodic functions is mostly $\mathrm{Bur}$. It is easy to see that $\mathcal{W} \mathcal{A P}(S)$ is a $C^{*}$-subalgebra of $\mathcal{C}_{\mathrm{b}}(S)$ whose character space we denote by $S_{\mathcal{W} \mathcal{A P}}$. It is clear that $S_{\mathcal{W A P}}$ contains a canonical, dense image of $S$. The multiplication of $S$ "extends" to $S_{\mathcal{W A} \mathcal{P}}$, turning it into a compact, semitopological semigroup. For more on semigroup compactifications, see [B-J-M]. The dual space $\mathcal{W} \mathcal{A} \mathcal{P}(S)^{*}$ can be identified with $M\left(S_{\mathcal{W A P}}\right)$, and thus, in particular, becomes a dual Banach algebra. For an alternative definition of the multiplication on $\mathcal{W} \mathcal{A} \mathcal{P}(S)^{*}$, see Pat, (2.8) Corollary and (2.11) Proposition].

From now on, we shall only consider weakly almost periodic functions on locally compact groups. If $G$ is a locally compact group, $\mathcal{C}_{0}(G) \subset \mathcal{W} \mathcal{A P}(G)$ holds and the canonical map from $G$ to $G_{\mathcal{W A P}}$ is a homeomorphism onto its image (Bur, Theorem 3.6]). It is straightforward that $G_{\mathcal{W A P}} \backslash G$ is a closed ideal of $G_{\mathcal{W} \mathcal{A P}}$ and thus, in particular, is a compact, semitopological semigroup. Let $\pi_{0}: \mathcal{W} \mathcal{A} \mathcal{P}(G)^{*} \rightarrow M(G)$ be the restriction map from $\mathcal{W} \mathcal{A P}(G)^{*}$ onto $\mathcal{C}_{0}(G)^{*}$. It is routinely checked that $\pi_{0}$ is a $w^{*}$-continuous algebra homomorphism. Consequently, $\mathcal{C}_{0}(G)^{\perp}=\operatorname{ker} \pi_{0}$ is a $w^{*}$-closed ideal in $\mathcal{W} \mathcal{A P}(G)^{*}$ that can be identified, as a Banach algebra, with $M\left(G_{\mathcal{W A P}} \backslash G\right)$.

As a corollary of Proposition 2.1, we obtain:

Corollary 2.3. Let $G$ be a locally compact group. Then the following are equivalent:

(i) The ideal $\mathcal{C}_{0}(G)^{\perp}$ of $\mathcal{W} \mathcal{A P}(G)^{*}$ has an identity.

(ii) The ideal $G_{\mathcal{W A P}} \backslash G$ of $G_{\mathcal{W A P}}$ has an identity.

Proof. All that needs to be shown is that, if $\mathcal{C}_{0}(G)^{\perp}$ has an identity element $\epsilon$, then $\|\epsilon\|=1$ must hold.

Denote the bimodule module action of $\mathcal{W} \mathcal{A P}(G)^{*}$ on $\mathcal{W} \mathcal{A P}(G)$ by $\cdot$. Let $f \in$ $\mathcal{W} \mathcal{A} \mathcal{P}(G)$ and observe that

$$
\begin{aligned}
|\langle f, \epsilon\rangle| & \leq \sup \left\{\left|\left\langle L_{x} f, \epsilon\right\rangle\right|: x \in G\right\} \\
& =\sup \left\{\left|\left\langle f, \delta_{x} * \epsilon\right\rangle\right|: x \in G\right\} \\
& =\sup \left\{\left|\left\langle\epsilon \cdot f, \delta_{x}\right\rangle\right|: x \in G\right\} \\
& =\|\epsilon \cdot f\| .
\end{aligned}
$$


Since $\epsilon \in \mathcal{C}_{0}(G)^{\perp}$, the left-hand side of (3) only depends on the equivalence class $\tilde{f}$ of $f$ in $\mathcal{W} \mathcal{A P}(G) / \mathcal{C}_{0}(G) \cong \mathcal{C}\left(G_{\mathcal{W} \mathcal{A P}} \backslash G\right)$. We thus obtain:

$$
\begin{aligned}
|\langle\tilde{f}, \epsilon\rangle| & \leq\|\epsilon \cdot \tilde{f}\| \\
& =\sup \left\{\left|\left\langle\epsilon \cdot \tilde{f}, \delta_{s}\right\rangle\right|: s \in G_{\mathcal{W A P}} \backslash G\right\} \\
& =\sup \left\{\left|\left\langle\tilde{f}, \delta_{s} * \epsilon\right\rangle\right|: s \in G_{\mathcal{W A P}} \backslash G\right\} \\
& =\sup \left\{\left|\left\langle\tilde{f}, \delta_{s}\right\rangle\right|: s \in G_{\mathcal{W A P}} \backslash G\right\} \\
& =\|\tilde{f}\| .
\end{aligned}
$$

Hence, $\|\epsilon\| \leq 1$ holds, which completes the proof.

In view of Corollary 2.3, we now turn to the question of whether, for a locally compact group $G$, the ideal $G_{\mathcal{W A P}} \backslash G$ can have an identity.

For any locally compact group $G$, let $G_{\mathcal{L U C}}$ denote its $\mathcal{L U C}$-compactification (see $[\mathrm{B}-\mathrm{J}-\mathrm{M}]$ ). There is a canonical quotient map $\pi: G_{\mathcal{L U C}} \rightarrow G_{\mathcal{W A P}}$. An element $s \in G_{\mathcal{L U C}}$ is called a point of unicity if $\pi^{-1}(\{\pi(s)\})=\{s\}$.

Recall that a locally compact group is called a [SIN]-group if its identity has a basis of neighborhoods invariant under conjugation; all abelian, all compact, and all discrete groups are [SIN]-groups.

The following is (mostly) [F-St, Theorem 1.4]:

Theorem 2.4. Let $G$ be a non-compact [SIN]-group. Then $G_{\mathcal{L U C}} \backslash G$ contains a dense open subset $X$ consisting of points of unicity with the following properties:

(i) $\pi(X)$ is open in $G_{\mathcal{W A P}} \backslash G$;

(ii) $X$ is invariant under multiplication with elements from $G$;

(iii) $X$ has empty intersection with $\left(G_{\mathcal{L U C}} \backslash G\right)^{2}$.

Remark. Items (ii) and (iii) are not explicitly stated as a part of $\mathrm{F}-\mathrm{St}$, Theorem 1.4], but follow from an inspection of the proof.

The following consequence of Theorem 2.4 was pointed out to me by Dona Strauss.

Corollary 2.5. Let $G$ be a non-compact [SIN]-group. Then the ideal $G_{\mathcal{W A P}} \backslash G$ does not have an identity.

Proof. Assume towards a contradiction that $G_{\mathcal{W A P}} \backslash G$ does have an identity, say $e$. Let $X$ be a set as specified in Theorem 2.4 Since $\pi(X)$ is open in $G_{\mathcal{W A P}} \backslash G$, and since $G e$ is dense in $G_{\mathcal{W A P}} \backslash G$, it follows that $G e \cap \pi(X) \neq \varnothing$; from Theorem 2.4(ii), we conclude that $e \in \pi(X)$. Let $p \in X$ be such that $\pi(p)=e$. Since $\pi\left(p^{2}\right)=e^{2}=e$ and since $p$ is a point of unicity, it follows that $p^{2}=p$. This, however, contradicts Theorem 2.4(iii).

\section{Normal, Virtual diagonals for $\mathcal{W} \mathcal{A} \mathcal{P}(G)^{*}$}

Given a locally compact group $G$, let $\mathcal{S C}\left(G_{\mathcal{W A P}} \times G_{\mathcal{W A P}}\right)$ denote the bounded, separately continuous functions on $G_{\mathcal{W A P}} \times G_{\mathcal{W A P}}$. The space $\mathcal{S C}\left(G_{\mathcal{W A P}} \times G_{\mathcal{W A P}}\right)$ can be canonically identified with $\mathcal{B}_{\sigma}^{2}\left(\mathcal{W} \mathcal{A} \mathcal{P}(G)^{*}, \mathbb{C}\right)$ ([Run 3, Proposition 2.5]). 
In terms of $\mathcal{S C}\left(G_{\mathcal{W A P}} \times G_{\mathcal{W A P}}\right)$, the bimodule action of $\mathcal{W} \mathcal{A P}(G)^{*}$ on $\mathcal{B}_{\sigma}^{2}\left(\mathcal{W} \mathcal{A P}(G)^{*}, \mathbb{C}\right)$ is given by

$$
(\mu \cdot f)(s, t):=\int_{G_{\mathcal{W A P}}} f(s, t r) d \mu(r) \quad\left(s, t \in G_{\mathcal{W} \mathcal{A P}}\right)
$$

and

$$
(f \cdot \mu)(s, t):=\int_{G_{\mathcal{W A P}}} f(r s, t) d \mu(r) \quad\left(s, t \in G_{\mathcal{W A P}}\right)
$$

for $f \in \mathcal{S C}\left(G_{\mathcal{W} \mathcal{A P}} \times G_{\mathcal{W} \mathcal{A P}}\right)$ and $\mu \in \mathcal{W} \mathcal{A P}(G)^{*}$ (this is seen as in Run 3, Proposition 3.1]).

The verification of our first lemma in this section is routine:

Lemma 3.1. Let $G$ be a locally compact group, and let

$$
I:=\left\{f \in \mathcal{S C}\left(G_{\mathcal{W A P}} \times G_{\mathcal{W A P}}\right): f(s, \cdot) \in \mathcal{C}_{0}(G) \text { for all } s \in G_{\mathcal{W} \mathcal{A P}}\right\} .
$$

Then:

(i) $\mathcal{S C}\left(G_{\mathcal{W} \mathcal{A P}} \times G_{\mathcal{W} \mathcal{A P}}\right)$, equipped with the supremum norm, is a commutative $C^{*}$-algebra with identity;

(ii) $I$ is a closed ideal and a $\mathcal{W} \mathcal{A P}(G)^{*}$-submodule of $\mathcal{S C}\left(G_{\mathcal{W A P}} \times G_{\mathcal{W A P}}\right)$.

Let $\mathfrak{A}$ be a $C^{*}$-algebra, and let $I$ be a closed ideal of $\mathfrak{A}$. As is well known, the second dual $\mathfrak{A}^{* *}$ is a von Neumann algebra - the enveloping von Neumann algebra of $\mathfrak{A}$-containing $I^{* *}$ as a $w^{* *}$ closed ideal. The identity $P$ of $I^{* *}$ is a central projection in $\mathfrak{A}^{* *}$ such that $I^{* *}=P \mathfrak{A}^{* *}$.

We make use of these facts in the case where $\mathfrak{A}=\mathcal{S C}\left(G_{\mathcal{W} \mathcal{A P}} \times G_{\mathcal{W A P}}\right)$ for a locally compact group $G$ and $I$ is as in (4).

Lemma 3.2. Let $G$ be a locally compact group, let $I$ be as in (4), and let $P \in$ $\mathcal{S C}\left(G_{\mathcal{W} \mathcal{A P}} \times G_{\mathcal{W A P}}\right)^{* *}$ be the identity of $I^{* *}$. Then $P \cdot \delta_{s}=P$ holds for all $s \in$ $G_{\mathcal{W} \mathcal{A P}}$.

Proof. For convenience, set $\mathfrak{A}:=\mathcal{S C}\left(G_{\mathcal{W} \mathcal{A P}} \times G_{\mathcal{W} \mathcal{A P}}\right)$, and let $\Omega$ be the character space of $\mathfrak{A}$, so that $\mathfrak{A} \cong \mathcal{C}(\Omega)$ via the Gelfand transform. Through point evaluation,

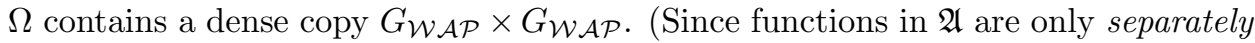
continuous on $G_{\mathcal{W} \mathcal{A P}} \times G_{\mathcal{W} \mathcal{A P}}$, the canonical map from $G_{\mathcal{W} \mathcal{A P}} \times G_{\mathcal{W} \mathcal{A P}}$ into $\Omega$ need not be continuous.) Since $I$ is a closed ideal of $\mathfrak{A}$, there is an open subset $U$ of $\Omega$ such that $I \cong \mathcal{C}_{0}(U)$. Point evaluation maps $G_{\mathcal{W A P}} \times G$ onto a dense subset of $U$.

We claim that $U$ is dense in $\Omega$. Since $G_{\mathcal{W A P}} \times G_{\mathcal{W A P}}$ is dense in $\Omega$, it is sufficient to show that each point in $G_{\mathcal{W A P}} \times G_{\mathcal{W} \mathcal{A P}}$ can be approximated by a net from $U$. Fix $(s, t) \in G_{\mathcal{W} \mathcal{A P}} \times G_{\mathcal{W} \mathcal{A P}}$, and let $f \in \mathfrak{A}$. Since $G$ is dense in $G_{\mathcal{W} \mathcal{A P}}$, there is a net $\left(x_{\alpha}\right)_{\alpha}$ in $G$ such that $x_{\alpha} \rightarrow t$; since $f(s, \cdot)$ is continuous, we have $f\left(s, x_{\alpha}\right) \rightarrow f(s, t)$; and since $f \in \mathfrak{A}$ is arbitrary, this yields that $\left(s, x_{\alpha}\right) \rightarrow(s, t)$ in $\Omega$.

Since $U$ is dense in $\Omega$, the ideal $I$ is essential in $\mathfrak{A}$, i.e. the only $f \in \mathfrak{A}$ such that $f I=\{0\}$ is $f=0$. By the universal property of the multiplier algebra, $\mathfrak{A}$ thus canonically embeds into $\mathcal{M}(I) \cong \mathcal{C}_{\mathrm{b}}(U)$, the multiplier algebra of $I$, which, in turn, can be identified with the idealizer of $I$ in $I^{* *}:\left\{F \in I^{* *}: F I \subset I\right\}$ (for all this, see Ped, for instance).

All in all, we have a canonical, injective ${ }^{*}$-homomorphism $\theta: \mathcal{C}(\Omega) \rightarrow I^{* *}$ with $\theta(\mathcal{C}(\Omega)) \subset \mathcal{M}(I)$, which is routinely seen to satisfy

$$
\theta\left(f \cdot \delta_{s}\right)=\theta(f) \cdot \delta_{s} \quad\left(f \in \mathfrak{A}, s \in G_{\mathcal{W A P}}\right)
$$


(just test both sides against points in $G_{\mathcal{W} \mathcal{A} \mathcal{P}} \times G$ ). Since clearly $\theta(1)=P$, we finally obtain that

$$
P \cdot \delta_{s}=\theta(1) \cdot \delta_{s}=\theta\left(1 \cdot \delta_{s}\right)=\theta(1)=P \quad\left(s \in G_{\mathcal{W A P}}\right) .
$$

This completes the proof.

Since $\mathcal{S C}\left(G_{\mathcal{W A P}} \times G_{\mathcal{W} \mathcal{A P}}\right)^{* *}$ is a von Neumann algebra for any locally compact group $G$, it follows that the canonical bimodule action of the commutative $C^{*}$-algebra $\mathcal{S C}\left(G_{\mathcal{W A P}} \times G_{\mathcal{W A P}}\right)$ on its dual extends to a bimodule action of $\mathcal{S C}\left(G_{\mathcal{W A P}} \times G_{\mathcal{W A P}}\right)^{* *}$ on its predual $\mathcal{S C}\left(G_{\mathcal{W A P}} \times G_{\mathcal{W A P}}\right)^{*}$. We denote this module action by mere juxtaposition.

Lemma 3.3. Let $G$ be a locally compact group, let $I$ be defined as in (4), and let $P$ denote the identity of $I^{* *}$. Then

$$
\left.\delta_{s} \cdot(P \mathrm{~N})=P\left(\delta_{s} \cdot \mathrm{N}\right) \quad\left(s \in G_{\mathcal{W A P}}\right)\right)
$$

holds for all $\mathrm{N} \in \mathcal{B}_{\sigma}^{2}\left(\mathcal{W} \mathcal{A P}(G)^{*}, \mathbb{C}\right)^{*}$.

Proof. First note that

$$
(f g) \cdot \delta_{s}=\left(f \cdot \delta_{s}\right)\left(g \cdot \delta_{s}\right) \quad\left(s \in G_{\mathcal{W A P}}\right)
$$

for all $f, g \in \mathcal{S C}\left(G_{\mathcal{W} \mathcal{A P}} \times G_{\mathcal{W} \mathcal{A P}}\right)$. By separate $w^{*}$-continuity, it follows that (5) holds as well for all $f, g \in \mathcal{S C}\left(G_{\mathcal{W A P}} \times G_{\mathcal{W} \mathcal{A P}}\right)^{* *}$.

Fix $s \in G_{\mathcal{W} \mathcal{A P}}$ and $\mathrm{N} \in \mathcal{B}_{\sigma}^{2}\left(\mathcal{W} \mathcal{A} \mathcal{P}(G)^{*}, \mathbb{C}\right)^{*}$, and let $f \in \mathcal{S C}\left(G_{\mathcal{W} \mathcal{A P}} \times G_{\mathcal{W} \mathcal{A P}}\right)$. We obtain

$$
\begin{aligned}
\left\langle f, \delta_{s} \cdot(P \mathrm{~N})\right\rangle & =\left\langle f \cdot \delta_{s}, P \mathrm{~N}\right\rangle \\
& =\left\langle\left(f \cdot \delta_{s}\right) P, \mathrm{~N}\right\rangle \\
& =\left\langle\left(f \cdot \delta_{s}\right)\left(P \cdot \delta_{s}\right), \mathrm{N}\right\rangle, \quad \text { by Lemma 3.2, } \\
& =\left\langle(f P) \cdot \delta_{s}, \mathrm{~N}\right\rangle, \quad \text { by (5) }, \\
& =\left\langle f P, \delta_{s} \cdot \mathrm{N}\right\rangle \\
& =\left\langle f, P\left(\delta_{s} \cdot \mathrm{N}\right)\right\rangle .
\end{aligned}
$$

This proves the claim.

Lemma 3.4. Let $G$ be a locally compact group, such that $\mathcal{W} \mathcal{A P}(G)^{*}$ has a normal, virtual diagonal, say $\mathrm{M}$, let $I$ be as in (4), and let $P$ be the identity of $I^{* *}$. Define

$$
\rho: \mathcal{W} \mathcal{A} \mathcal{P}(G)^{*} \rightarrow \mathcal{W} \mathcal{A} \mathcal{P}(G)^{*}, \quad \mu \mapsto \Delta_{\sigma}(P(\mathrm{M} \cdot \mu)) .
$$

Then:

(i) $\mathcal{C}_{0}(G)^{\perp}$ is contained in $\operatorname{ker} \rho$;

(ii) $\pi_{0} \circ \rho=\pi_{0}$ holds, where $\pi_{0}: \mathcal{W} \mathcal{A} \mathcal{P}(G)^{*} \rightarrow M(G)$ is the canonical restriction map;

(iii) $\rho\left(\delta_{s} * \mu\right)=\delta_{s} * \rho(\mu)$ for all $s \in G_{\mathcal{W A P}}$ and $\mu \in \mathcal{W} \mathcal{A P}(G)^{*}$.

Proof. (i): Let $\mu \in \mathcal{C}_{0}(G)^{\perp}$. It follows that $\mu \cdot f=0$ for each $f \in I$ and, consequently, that $\mathrm{M} \cdot \mu \in I^{\perp}$. Let $F \in \mathcal{S C}\left(G_{\mathcal{W} \mathcal{A P}} \times G_{\mathcal{W A P}}\right)^{* *}$, and note that $F P \in I^{* *}$. Since

$$
\langle F, P(\mathrm{M} \cdot \mu)\rangle=\langle F P, \mathrm{M} \cdot \mu\rangle=0,
$$

we conclude that $\mu \in \operatorname{ker} \rho$. 
(ii): Fix $\mu \in \mathcal{W} \mathcal{A P}(G)^{*}$ and $f \in \mathcal{C}_{0}(G)$, and observe that

$$
\begin{aligned}
\langle f, \rho(\mu)\rangle & =\left\langle f, \Delta_{\sigma}(P(\mathrm{M} \cdot \mu))\right\rangle \\
& =\left\langle\Delta^{*} f, P(\mathrm{M} \cdot \mu)\right\rangle \\
& =\left\langle\left(\Delta^{*} f\right) P, \mathrm{M} \cdot \mu\right\rangle \\
& =\left\langle\Delta^{*} f, \mathrm{M} \cdot \mu\right\rangle, \quad \text { because } \Delta^{*} \mathcal{C}_{0}(G) \subset I, \\
& =\left\langle f, \Delta_{\sigma}(\mathrm{M} \cdot \mu)\right\rangle \\
& =\langle f, \mu\rangle .
\end{aligned}
$$

This proves the claim.

(iii): Fix $\mu \in \mathcal{W} \mathcal{A P}(G)^{*}$ and $s \in G_{\mathcal{W} \mathcal{A P}}$. We obtain

$$
\begin{array}{rlrl}
\rho\left(\delta_{s} * \mu\right) & =\Delta_{\sigma}\left(P\left(\left(\mathrm{M} \cdot \delta_{s}\right) \cdot \mu\right)\right) & \\
& =\Delta_{\sigma}\left(P\left(\delta_{s} \cdot(\mathrm{M} \cdot \mu)\right)\right), & & \text { by Definition 1.3, } \\
& =\Delta_{\sigma}\left(\delta_{s} \cdot(P(\mathrm{M} \cdot \mu))\right), & & \text { by Lemma 3.3. } \\
& =\delta_{s} * \Delta_{\sigma}(P(\mathrm{M} \cdot \mu)) & \\
& =\delta_{s} * \rho(\mu) .
\end{array}
$$

This completes the proof.

We can now prove the main result of this section (and of the whole paper):

Theorem 3.5. Let $G$ be a non-compact [SIN]-group. Then $\mathcal{W} \mathcal{A P}(G)^{*}$ does not have a normal, virtual diagonal.

Proof. Assume towards a contradiction that $\mathcal{W} \mathcal{A} \mathcal{P}(G)^{*}$ has a normal, virtual diagonal. Let $\rho: M(G) \rightarrow \mathcal{W} \mathcal{A P}(G)^{*}$ be as in Lemma 3.4, and define $\epsilon:=\delta_{e}-\rho\left(\delta_{e}\right)$, where $e$ is the identity of $G$. By Lemma 3.4(ii), it is clear that $\epsilon \in \mathcal{C}_{0}(G)^{\perp}$. Moreover, we have for $s \in G_{\mathcal{W A P}} \backslash G$ that

$$
\begin{aligned}
\delta_{s} * \epsilon & =\delta_{s}-\delta_{s} * \rho\left(\delta_{e}\right) \\
& =\delta_{s}-\rho\left(\delta_{s} * \delta_{e}\right), \quad \text { by Lemma 3.4(iii), } \\
& =\delta_{s}, \quad \text { by Lemma 3.4(i). }
\end{aligned}
$$

Consequently, $\epsilon$ is a right identity for $\mathcal{C}_{0}(G)^{\perp}$.

For any $f \in \mathcal{W} \mathcal{A} \mathcal{P}(G)$, the function $\check{f}: G \rightarrow \mathbb{C}$ defined by letting $\check{f}(x):=f\left(x^{-1}\right)$ for $x \in G$ lies also in $\mathcal{W} \mathcal{A} \mathcal{P}(G)$ ([Bur, Corollary 1.18]). Setting

$$
\langle f, \check{\mu}\rangle:=\langle\check{f}, \mu\rangle \quad\left(\mu \in \mathcal{W} \mathcal{A} \mathcal{P}(G)^{*}, f \in \mathcal{W} \mathcal{A} \mathcal{P}(G)\right)
$$

defines an anti-automorphism $\mathcal{W} \mathcal{A P}(G)^{*} \ni \mu \mapsto \check{\mu}$ of $\mathcal{W} \mathcal{A P}(G)^{*}$, which leaves $\mathcal{C}_{0}(G)^{\perp}$ invariant. Hence, $\check{\epsilon}$ is a left identity for $\mathcal{C}_{0}(G)^{\perp}$, so that $\mathcal{C}_{0}(G)^{\perp}$ has in fact an identity. This, however, is not possible by Corollaries 2.3 and 2.5 .

The following corollary confirms the guess made at the end of [Run 5] for [SIN]group.

Corollary 3.6. Consider the following statements about a [SIN]-group $G$ :

(i) $\mathcal{W} \mathcal{A P}(G)^{*}$ has a normal, virtual diagonal.

(ii) $G$ is compact.

(iii) $G$ is amenable.

(iv) $\mathcal{W} \mathcal{A P}(G)^{*}$ is Connes-amenable. 
Then
(i) $\Longleftrightarrow$
(ii) $\Longrightarrow$
(iii)
(iv).

Proof. (i) $\Longrightarrow$ (ii) follows immediately from Theorem 3.5, and the converse is shown in Run 4 (for compact $G$, we have $\mathcal{W} \mathcal{A} \mathcal{P}(G)^{*}=M(G)$ ).

(ii) $\Longrightarrow$ (iii) is well known (see [Pat] or [Run 2, Chapter 1]).

(iii) $\Longleftrightarrow$ (iv) is [Run 5, Proposition 4.11].

Consequently, $\mathcal{W} \mathcal{A} \mathcal{P}(G)^{*}$ is a Connes-amenable dual Banach algebra without a normal, virtual diagonal whenever $G$ is an amenable, but not compact [SIN]-group: this includes all non-compact, abelian, locally compact groups as well as all infinite, discrete, amenable groups.

As a consequence of Corollary [3.6, we also obtain a characterization of those locally compact groups $G$ for which $\mathcal{W} \mathcal{A P}(G)^{*}$ is amenable (in the sense of [Joh 2]):

Corollary 3.7. The following are equivalent for a locally compact group $G$ :

(i) $\mathcal{W} \mathcal{A} \mathcal{P}(G)^{*}$ is amenable;

(ii) $G$ is finite.

Proof. Of course, only (i) $\Longrightarrow$ (ii) needs proof.

If $\mathcal{W} \mathcal{A P}(G)^{*}$ is amenable, so is its quotient $M(G)$. By [D-G-H], this means that $G$ must be discrete. In particular, $G$ is a [SIN]-group. Since $\mathcal{W} \mathcal{A P}(G)^{*}$ is amenable, it must have a normal, virtual diagonal, so that the discrete group $G$ is also compact by Corollary 3.6 .

\section{Minimally Weakly almost periodic groups}

In view of Corollary [3.6, the conjecture (made in Run 5]) is tempting that $\mathcal{W} \mathcal{A} \mathcal{P}(G)^{*}$ has a normal, virtual diagonal only if $G$ is compact. As we shall see in this final section, this is wrong.

Recall that a continuous, bounded function $f$ on a locally compact group $G$ is called almost periodic if $\left\{L_{x} f: x \in G\right\}$ is relatively compact in the norm topology of $\mathcal{C}_{\mathrm{b}}(G)$. Let

$$
\mathcal{A P}(G):=\left\{f \in \mathcal{C}_{\mathrm{b}}(G): f \text { is almost periodic }\right\} .
$$

Like $\mathcal{W} \mathcal{A} \mathcal{P}(G)$, the space $\mathcal{A P}(G)$ is a commutative $C^{*}$-algebra. Its character space, denoted by $G_{\mathcal{A P}}$, is a compact group that contains a dense, but generally not homeomorphic image of $G$ in a canonical manner. For more information, see [Bur] or $[\mathrm{B}-\mathrm{J}-\mathrm{M}$, for example.

The following definition is from Chou 1]:

Definition 4.1. Let $G$ be a locally compact group. Then $G$ is called minimally weakly almost periodic if $\mathcal{W} \mathcal{A} \mathcal{P}(G)=\mathcal{A P}(G)+\mathcal{C}_{0}(G)$.

Remarks. 1. Every compact group is trivially minimally weakly almost periodic. If $G$ is not compact, but weakly almost periodic, then the sum in Definition 4.1 is a direct one.

2. The motion group $\mathbb{R}^{N} \rtimes \mathrm{SO}(N)$ is minimally weakly almost periodic (and amenable) as is $\operatorname{SL}(2, \mathbb{R})$, which is not amenable (see [Chou 1]).

3. If $G$ is minimally weakly almost periodic, the kernel of $G_{\mathcal{W A P}}$ (see $\mathrm{Bur}$ for the definition) must equal $G_{\mathcal{W} \mathcal{A P}} \backslash G$. Hence, by Corollary 2.3, a non-compact [SIN]group cannot be minimally weakly almost periodic. (This follows also immediately from the main result of [Chou 2]). 
The verification of the following lemma is routine.

Lemma 4.2. Let $\mathfrak{A}$ and $\mathfrak{B}$ be dual Banach algebras each of which as a normal, virtual diagonal. Then $\mathfrak{A} \oplus \mathfrak{B}$ has a normal, virtual diagonal.

It is now fairly straightforward to refute our "conjecture" from [Run 5]:

Proposition 4.3. Let $G$ be a locally compact, minimally weakly almost periodic group. Then the following are equivalent:

(i) $G$ is amenable.

(ii) $\mathcal{W} \mathcal{A} \mathcal{P}(G)^{*}$ has a normal, virtual diagonal.

Proof. (i) $\Longrightarrow$ (ii): Without loss of generality, suppose that $G$ is not compact. Let $\pi_{a}: \mathcal{W} \mathcal{A P}(G)^{*} \rightarrow \mathcal{A P}(G)^{*}$ and $\pi_{0}: \mathcal{W} \mathcal{A P}(G)^{*} \rightarrow M(G)$ be the respective restriction maps; they are $w^{*}$-continuous algebra homomorphism. Since $\mathcal{W} \mathcal{A} \mathcal{P}(G)=$ $\mathcal{A P}(G) \oplus \mathcal{C}_{0}(G)$, it follows that $\pi_{a} \oplus \pi_{0}: \mathcal{W} \mathcal{A P}(G)^{*} \rightarrow \mathcal{A P}(G)^{*} \oplus M(G)$ is a $w^{*}$ continuous isomorphism. Since $G$ is amenable $M(G)$ has a normal, virtual diagonal by Run 4, and the same is true for $\mathcal{A P}(G)^{*} \cong M\left(G_{\mathcal{A P}}\right)$. From Lemma 4.2, we conclude that $\mathcal{W} \mathcal{A} \mathcal{P}(G)^{*}$ has a normal, virtual diagonal.

(ii) $\Longrightarrow$ (i) follows immediately from [Run 5, Proposition 4.11].

Example. The motion group group $G:=\mathbb{R}^{N} \rtimes \mathrm{SO}(N)$ is minimally weakly almost periodic and amenable, so that $\mathcal{W} \mathcal{A P}(G)^{*}$ has a normal, virtual diagonal even though $G$ fails to be compact.

In view of Proposition 4.3 and Corollary 3.6, the conjecture is not so farfetched that $\mathcal{W} \mathcal{A P}(G)^{*}$ has a normal, virtual diagonal if and only if $G$ is amenable and minimally weakly almost periodic.

\section{ACKNOWLEDGMENT}

I am grateful to Dona Strauss of Hull for bringing $[\mathrm{F}-\mathrm{St}$ ] to my attention and for providing Corollary 2.3 and its proof.

\section{REFERENCES}

[B-J-M] J. F. Berglund, H. D. Junghenn, and P. Milnes, Analysis on Semigroups. WileyInterscience, 1988. MR0999922 (91b:43001)

[B-P] J. W. Bunce and W. L. PASchKe, Quasi-expectations and amenable von Neumann algebras. Proc. Amer. Math. Soc. 71 (1978), 232-236. MR0482252 (58:2330)

[Bur] R. B. Burckel, Weakly Almost Periodic Functions on Semigroups. Gordon and Breach, 1970. MR0263963 (41:8562)

[Chou 1] C. Chou, Minimally weakly almost periodic groups. J. Funct. Anal. 36 (1980), 1-17. MR0568972 (81f:43009)

[Chou 2] C. CHOU, Weakly almost periodic functions and Fourier-Stieltjes algebras of locally compact groups. Trans. Amer. Math. Soc. 274 (1982), 141-157. MR0670924 (84a:43008)

[Con 1] A. Connes, Classification of injective factors. Ann. of Math. 104 (1976), 73-114. MR:0454659 (56:12908)

[Con 2] A. Connes, On the cohomology of operator algebras. J. Funct. Anal. 28 (1978), 248253. MR $0493383(58: 12407)$

[C-G] G. CORACH and J. E. GalÉ, Averaging with virtual diagonals and geometry of representations. In: E. Albrecht and M. Mathieu (eds.), Banach Algebras '97, pp. 87-100. Walter de Grutyer, 1998. MR1656600 (99m:46167)

[D-G-H] H. G. Dales, F. Ghahramani, and A. Ya. HelemskiĬ, The amenability of measure algebras. J. London Math. Soc. (2) 66 (2002), 213-226. MR1911870(2003c:43001)

[Eff] E. G. Effros, Amenability and virtual diagonals for von Neumann algebras. J. Funct. Anal. 78 (1988), 137-156. MR0937636 (89e:46072) 
[E-L] E. G. Effros and E. C. Lance, Tensor products of operator algebras. J. Funct. Anal. 25 (1977), 1-34. MR0448092 (56:6402)

[E-K] E. G. Effros and A. Kishimoto, Module maps and Hochschild-Johnson cohomology. Indiana Univ. Math. J. 36 (1987), 257-276. MR0891774 (89b:46068)

[F-St] S. Ferri and D. Strauss, A note on the $\mathcal{W} \mathcal{A} \mathcal{P}$-compactification and the $\mathcal{L U C}$-compactification of a topological group. Semigroup Forum 69 (2004), 87-101. MR2063981

[Hel] A. YA. HeLEMSKII , Homological essence of amenability in the sense of A. Connes: the injectivity of the predual bimodule (translated from the Russian). Math. USSR-Sb 68 (1991), 555-566. MR1038222(91f:46102)

[Joh 1] B. E. Johnson, Separate continuity and measurability. Proc. Amer. Math. Soc. 20 (1969), 420-422. MR0236345 (38:4641)

[Joh 2] B. E. Johnson, Cohomology in Banach algebras. Mem. Amer. Math. Soc. 127 (1972). MR.0374934(51:11130)

[Joh 3] B. E. Johnson, Approximate diagonals and cohomology of certain annihilator Banach algebras. Amer. J. Math. 94 (1972), 685-698. MR0317050 (47:5598)

[J-K-R] B. E. Johson, R. V. Kadison, and J. Ringrose, Cohomology of operator algebras, III. Bull. Soc. Math. France 100 (1972), 73-79. MR0318908 (47:7454)

[Pat] A. L. T. Paterson, Amenability. American Mathematical Society, 1988. MR0961261 (90e:43001)

[Ped] G. K. Pedersen, C*-Algebras and their Automorphism Groups. Academic Press, 1979. MR 0548006 (81e:46037)

[Run 1] V. Runde, Amenability for dual Banach algebras. Studia Math. 148 (2001), 47-66. MR:1881439 (2002m:46078)

[Run 2] V. Runde, Lectures on Amenability. Lecture Notes in Mathematics 1774, Springer Verlag, 2002. MR.1874893(2003h:46001)

[Run 3] V. Runde, Connes-amenability and normal, virtual diagonals for measure algebras, I. J. London Math. Soc. 67 (2003), 643-656. MR.1967697(2004c:43003)

[Run 4] V. Runde, Connes-amenability and normal, virtual diagonals for measure algebras, II. Bull. Austral. Math. Soc. 68 (2003), 325-328. MR2016307(2004j:43002)

[Run 5] V. Runde, Dual Banach algebras: Connes-amenability, normal, virtual diagonals, and injectivity of the predual bimodule. Math. Scand. 95 (2004), 124-144. MR2091485

[Was 1] S. Wassermann, On Tensor products of certain group $C^{*}$-algebras. J. Funct. Anal. 23 (1976), 239-254. MR0425628 (54:13582)

[Was 2] S. Wassermann, Injective $W^{*}$-algebras. Math. Proc. Cambridge Phil. Soc. 82 (1977), 39-47. MR0448108(56:6418)

Department of Mathematical and Statistical Sciences, University of Alberta, Edmonton, Alberta, Canada T6G 2G1

E-mail address: vrunde@ualberta.ca

$U R L:$ http://www.math.ualberta.ca/ runde/ 